Olga G Falceto'

Carmen L Fernandes"

Claudia Baratojo"'I

Elsa R J Giugliani'v

Departamento de Psiquiatria e Medicina Legal. Faculdade de Medicina (FM). Universidade Federal do Rio Grande do Sul (UFRGS). Porto Alegre, RS, Brasil

Gerência de Medicina de Saúde e Comunidade. Grupo Hospitalar Nossa Senhora da Conceição. Porto Alegre, RS, Brasil

III Programa de Pós-Graduação em Ciências Médicas. FM - UFRGS. Porto Alegre, RS, Brasil

Iv Departamento de Pediatria e Puericultura. FM - UFRGS. Porto Alegre, RS, Brasil

Correspondência | Correspondence: Olga Garcia Falceto

Departamento de Psiquiatria e Medicina Legal Faculdade de Medicina da UFRGS

R. Ramiro Barcelos, 2350

90035-003 Porto Alegre, RS, Brasil

E-mail: olgafalceto@uol.com.br

\section{Fatores associados ao envolvimento do pai nos cuidados do lactente}

\section{Factors associated with father involvement in infant care}

\section{RESUMO}

OBJETIVO: Identificar fatores associados à falta de envolvimento ativo do pai nos cuidados de crianças aos quatro meses.

MÉTODOS: Estudo transversal envolvendo famílias de 153 crianças aos quatro meses de vida, entrevistadas em suas casas por dois terapeutas de famílias em Porto Alegre (RS), 1998-2000. Estudo transversal envolvendo famílias de 153 crianças aos quatro meses de vida, entrevistadas em suas casas por dois terapeutas de famílias. Além do envolvimento do pai nos cuidados do lactente foram examinadas características sociodemográficas, saúde mental dos pais (utilizando a escala Self Report Questionnaire-20 e avaliação com os critérios do Diagnostic and Statistical Manual of Mental Disorders-IV) e qualidade do relacionamento conjugal (usando a escala Global Assessment of Relational Functioning do Diagnostic and Statistical Manual of Mental Disorders-IV). Utilizou-se regressão de Poisson para avaliar a associação entre falta de envolvimento do pai nos cuidados do filho e variáveis selecionadas. A magnitude das associações foi estimada pela razão de prevalências.

RESULTADOS: Os pais de 13\% dos lactentes não tinham qualquer contato com seus filhos. Entre as famílias em que os pais coabitavam (78\% do total), $33 \%$ dos pais relataram não participar ativamente nos cuidados de seus filhos. Relação conjugal problemática e mãe ser “do lar” mostraram-se associadas à falta de envolvimento dos pais nos cuidados do filho.

CONCLUSÕES: É alta a prevalência de famílias nas quais o pai não tem envolvimento ativo no cuidado de seu filho, ocorrendo em especial quando a relação conjugal é problemática e a mãe não tem trabalho remunerado

DESCRITORES: Paternidade. Comportamento Paterno. Relações PaiFilho. Relações Familiares. Cuidado da Criança. Estudos Transversais. 


\begin{abstract}
OBJECTIVE: To identify factors associated with the lack of active father involvement in infant care at four months of age.

METHODS: Cross-sectional study involving families of 153 infants at four months of age, interviewed in their homes by two family therapists in the city of Porto Alegre (Southern Brazil), 1998-2000. In addition. Cross-sectional study involving families of 153 infants at four months of age, interviewed in their homes by two family therapists. In addition to father involvement in infant care, sociodemographic, parental mental health (using the Self Report Questionnaire-20 scale and Diagnostic and Statistical Manual of Mental Disorders-IV criteria assessment) and quality of couple relationship characteristics (using the Assessment of Relational Functioning from Diagnostic and Statistical Manual of Mental Disorders-IV) were analyzed. Poisson regression was employed to assess the association between lack of father involvement in child care and the variables selected. Prevalence ratio was used to estimate the magnitude of associations.
\end{abstract}

RESULTS: Fathers of $13 \%$ of infants had no contact with their children. Among families whose parents lived together (78\% of all), 33\% of the fathers reported not actively participating in their children's care. Problematic couple relationship and mother as a housewife were associated with lack of father involvement in infant care.

CONCLUSIONS: High prevalence of families whose father is not actively involved with infant care, especially when couple relationship is problematic and the mother does not have a paid job.

DESCRIPTORS: Paternity. Paternal Behavior. Father-Child Relations. Family Relations. Child Care. Cross-Sectional Studies.

\section{INTRODUÇÃO}

A ausência total ou relativa da figura paterna durante a infância tem sido associada com transtornos do desenvolvimento tais como problemas emocionais, níveis menos elevados de desenvolvimento cognitivo, abuso de drogas, transtornos de conduta, gravidez na adolescência, entre outros. ${ }^{1,7,21}$

No período anterior à década de 1960, ressaltava-se apenas o papel socioemocional do pai no processo de separação do vínculo simbiótico tido como normal entre mãe e filho e no estabelecimento de limites na educação, além de conseqüências negativas de sua ausência relativa ou total na saúde mental dos filhos. Na década de 1960, período em que se consolidava o movimento feminista, as publicações começaram a valorizar o envolvimento direto do pai no cuidado com os filhos. A participação do pai passou a ser considerada necessária e reivindicada pelas mães à medida em que essas se diferenciavam das gerações anteriores pelo engajamento progressivo em atividades laborais externas ao $l a{ }^{23}$ Freqüentemente tal participação passou a ser desejada pelo pai ao descobrir o prazer de compartilhar da intimidade de seus filhos.

Atualmente, estudos enfatizam as diferenças nos tipos de cuidados prestados pela mãe e pelo pai nos quais os pais passam menos tempo com os filhos e relacionamse com eles por meio de atividades e brincadeiras. Há consenso quanto à predominância da responsabilidade materna nos cuidados dos filhos, apesar das atividades profissionais da mulher. ${ }^{9}$

Algumas pesquisas observaram melhor desenvolvimento cognitivo do filho quando o pai está envolvido nos seus cuidados, uma vez que sua interação com a criança difere da mãe, com a introdução de novidades e desafios. ${ }^{11}$ Foram também observados maior competência social, menor índice de transtornos de conduta e melhor saúde mental das crianças que tiveram o cuidado direto do pai. ${ }^{1,7,21}$ Estudos comparativos sobre o tipo de vínculo da criança com o pai e a mãe sugerem que: 1 ) mulher e homem são igualmente capazes de desenvolver uma relação sensível e consistente com o filho; 2) o estilo de vínculo que a criança desenvolve em suas relações interpessoais é influenciado pelo estilo de sua relação com o pai, mas é principalmente associado à relação que ela tem com sua mãe; 3) o tipo de relação do pai com a criança está altamente associado com o tipo de relação dessa com a mãe; 4) o envolvimento do pai nos cuidados está associado com a criança apresentar melhor 
capacidade de empatia, desenvolvimento cognitivo e relacionamento com outras crianças, independentemente da relação que a criança tem com a mãe, 5) o desenvolvimento da capacidade de regular as emoções parece estar mais associado com as experiências paifilho do que mãe-filho. ${ }^{12,16,20}$

Não foram identificados estudos longitudinais que definam os fatores de proteção e de risco para o estabelecimento e manutenção do envolvimento do pai nos cuidados dos filhos. Diante da importância do tema para o adequado desenvolvimento das crianças e da escassez de literatura brasileira sobre o tema, o presente estudo teve por objetivo identificar fatores associados à falta de envolvimento do pai nos cuidados do lactente.

\section{MÉTODOS}

$\mathrm{O}$ artigo aborda um aspecto de uma pesquisa mais ampla sobre o funcionamento familiar e sua relação com diversos desfechos relacionados com a saúde física e mental das crianças. Um dos objetivos da pesquisa era investigar fatores associados com o desmame precoce, definido como a interrupção do aleitamento materno antes dos quatro meses. Este ponto de corte foi utilizado por haver evidências científicas de que o desmame antes dos quatro meses está associado com diversos agravos à saúde da criança. ${ }^{10}$ Alguns resultados do estudo já foram publicados. ${ }^{4,5}$

A pesquisa foi realizada em um bairro de Porto Alegre (RS), que abriga aproximadamente 18.000 habitantes. O bairro é servido por três unidades de saúde do serviço de saúde comunitária, de onde surgiu a solicitação de uma pesquisa acadêmica que oferecesse subsídios para a elaboração de um protocolo para atender crianças de zero a cinco anos. A população da região é economicamente heterogênea, sendo a maioria de classe média baixa, com algumas famílias muito pobres. Todas as casas têm água encanada e eletricidade, mas em torno de $10 \%$ das residências não contam com rede de esgoto e coleta de lixo.

Para seleção da amostra foram utilizados os registros de nascimento em hospitais da cidade (Declaração de Nascidos Vivos da Secretaria Municipal de Saúde de Porto Alegre). Todas as famílias do bairro (256) que tinham lactentes de quatro meses nascidos entre novembro/1998 e fevereiro/2000 foram visitadas em seus domicílios e solicitadas a participar da pesquisa. Colheram-se dados de identificação; sete famílias recusaram-se. Estas foram consideradas perda somadas às 103 famílias que não foram encontradas em três tentativas de realizar-se a segunda visita.

As entrevistas foram realizadas por dois terapeutas de família capacitados para o diagnóstico e tratamento dos transtornos do relacionamento familiar. Os entrevistadores receberam treinamento específico e participaram da formulação final do questionário, sem contudo saberem os objetivos da pesquisa. O estudo piloto foi realizado com dez famílias. Durante a coleta de dados, as investigadoras principais realizaram entrevistas em dupla com diferentes entrevistadores para se certificarem da uniformidade das avaliações feitas pela equipe. A dupla de entrevistadores mudava a cada entrevista para evitar vieses de avaliação.

As entrevistas, com duração de cerca de duas horas, seguiram um roteiro semi-estruturado e foram filmadas por um estudante de medicina. Num primeiro momento, eram coletados dados sociodemográficos, obstétricos e sobre as características do lactente. Posteriormente, havia uma conversa inicial com todos os membros da família, seguida de entrevista com o casal e, finalmente, o pai e a mãe eram entrevistados individualmente. A coleta de informações foi organizada de forma a priorizar a aproximação com a família, facilitando o estabelecimento de vínculo que auxiliasse na fidelidade das informações. Simultaneamente, observava-se o tipo e a qualidade das relações interpessoais que ocorriam entre os membros da família.

Na entrevista, foram utilizados diferentes instrumentos para investigar as histórias pessoal, clínica e psiquiátrica dos cônjuges, a relação do casal, o nascimento e desenvolvimento do lactente e a relação dos pais com o lactente.

O envolvimento do pai nos cuidados do filho foi avaliado pelos relatos dos entrevistados sobre si próprios, seus cônjuges, suas relações e também pela observação direta, durante aproximadamente duas horas, da forma como o pai se comunicava e cuidava de seu filho. A categorização do envolvimento do pai foi feita previamente, baseada na experiência das autoras, sendo categorizado como: 1) ativamente envolvido: contato diário do pai com a criança e participação em atividades tais como consolar a criança, brincar com ela, alimentá-la, banhála, entre outras, seja espontaneamente ou a pedido da companheira; 2) sem participação direta porém apoiando a mãe: manifestação explícita de satisfação do pai com os cuidados que a mãe dava ao filho; 3) sem participação: pai emocional e fisicamente ausente dos cuidados, e 4) atrapalhando os cuidados do lactente: mãe negativamente influenciada pelo pai no seu desempenho de provedora de cuidados. Nas análises estatísticas, foram comparadas a categoria $1 \mathrm{com}$ a soma das categorias 2, 3 e 4 .

A relação do casal foi avaliada utilizando-se a escala GARF (Avaliação Global do Funcionamento Relacional, do DSM-IV). A pontuação da escala varia de 1 a 5 (unidade relacional avaliada desde satisfatória até excessivamente disfuncional para a continuidade de contato e ligação, segundo relato dos participantes e observação dos entrevistadores). Para efeitos de análise, a relação do casal foi categorizada em: "sem dificuldades importantes”, quando a pontuação alcançava 1 ou 2; e em "com dificuldades de moderadas a graves”, quando variava de 3 a 5 pontos. 
Na investigação da saúde mental, pai e mãe responderam, independentemente, o Self Report Questionnaire (SRQ), o qual identifica sintomas que perturbam o funcionamento no dia a dia, em especial depressão e ansiedade. Conforme validação desse instrumento no Brasil,${ }^{15}$ uma pontuação de sete ou mais para as mulheres e de cinco ou mais para os homens leva à suspeição diagnóstica de transtorno mental. Os entrevistadores também elaboravam uma impressão diagnóstica utilizando os critérios do DSM-IV.

Após cada entrevista, os dois entrevistadores discutiam suas avaliações e buscavam um consenso. As dúvidas eram discutidas com uma das pesquisadoras e, se necessário, examinava-se o vídeo da entrevista.

A dupla digitação dos dados com posterior comparação permitiu a correção de eventuais erros.

Para testar as associações e sua magnitude, utilizouse o teste do qui-quadrado com correção de Yates e a razão de prevalências (intervalo de confiança de 95\%). Posteriormente, foi realizada regressão de Poisson com estimativa de variâncias robustas, fazendo parte do modelo as variáveis que na análise bivariada estiveram associadas $(\mathrm{p}<0,2)$ com falta de envolvimento ativo do pai. O nível de significância estatística adotado foi de 0,05 . As análises foram feitas nos programas SPSS 12.0 e Stata 7.0 .

O projeto de pesquisa foi aprovado pelos Comitês de Ética em Pesquisa do Grupo Hospitalar Conceição e do Hospital de Clínicas de Porto Alegre. Todos os pais e mães assinaram termo de consentimento livre e esclarecido.

\section{RESULTADOS}

Dentre as 153 famílias localizadas, o pai não coabitava com a mãe e a criança em 34 famílias (11 tinham algum contato com o filho e apoiavam a mãe, 20 não tinham contato, dois haviam falecido e um se encontrava no sistema prisional) e em uma não foi possível observar o envolvimento do pai com o lactente. Assim, foram estudadas 118 famílias em que havia casal coabitando, o pai foi entrevistado e seu envolvimento com o lactente observado.

Inicialmente, foi realizada análise comparativa entre as famílias que completaram o estudo e aquelas que não foram entrevistadas, mas com dados de identificação, com o objetivo de verificar um possível viés de seleção. Foram estudadas variáveis do lactente e dos pais, verificandose um número significativamente maior de homens não brancos $(\mathrm{p}=0,016)$ e de lactentes de baixo peso ao nascer $(p=0,001)$ no grupo que não completou o estudo. Essas variáveis, no entanto, não mostraram associação com o envolvimento do pai nos cuidados do lactente.
O pai de $48,3 \%$ das crianças não se envolvia com elas diariamente. Entre as famílias em que os pais coabitavam, esse valor foi 33\% (Tabela 1). Nenhum pai parecia estar atrapalhando os cuidados prestados pela mãe à criança.

As Tabelas 2 e 3 apresentam os resultados das análises bivariadas. As seguintes variáves foram selecionadas para o modelo multivariado por mostrarem associação $(p<0,20)$ com o desfecho: renda familiar, cor da mãe, escolaridade do pai e da mãe, ocupação da mãe, sexo do lactente, suspeita de transtorno mental no pai e na mãe e relação conjugal com problemas de moderados a graves.

Foram identificados pela escala SRQ índices elevados de suspeita de transtorno mental, correspondendo a $16,1 \%$ nos pais e a $37,3 \%$ nas mães. Esses índices foram maiores segundo a avaliação clínica dos entrevistadores que identificaram sinais de depressão em $24,0 \%$ dos pais e em 36,8\% das mães. Ainda, 7,1\% dos pais e $8,8 \%$ das mães apresentavam ansiedade e $10,1 \%$ dos pais e $2,4 \%$ das mães mostravam sinais de uso abusivo de álcool. A proporção de casais em que ambos apresentavam algum transtorno correspondeu a $34,1 \%$ do total da amostra.

Relações conjugais com dificuldades de moderadas a graves corresponderam a $23,7 \%$ dos casais.

A análise multivariada de regressão de Poisson (Tabela 4) confirmou a significância estatística da associação entre ausência de envolvimento ativo do pai nos cuidados do lactente e as variáveis relação conjugal com dificuldades de moderadas a graves e mãe sem ocupação laboral fora do lar.

\section{DISCUSSÃO}

A importância do vínculo pai-lactente nos primeiros meses de vida é cada vez mais valorizada na sociedade. A exemplo do vínculo mãe-lactente, ${ }^{2}$ os primeiros meses de convivência são cruciais no estabelecimento da função paterna. Entretanto, esse assunto é ainda pouco abordado na literatura.

Tabela 1. Distribuição dos pais que coabitam com seus filhos conforme tipo de envolvimento nos cuidados. Porto Alegre, RS, 1998-2000.

\begin{tabular}{lcc}
\hline Variável & $\mathrm{n}$ & $\%$ \\
\hline Pai envolve-se ativamente & 79 & 67,0 \\
Pai apóia mas não participa & 34 & 28,8 \\
Pai emocionalmente ausente & 5 & 4,2 \\
Pai atrapalha nos cuidados & 0 & 0 \\
\hline Total & 118 & 100,0 \\
\hline
\end{tabular}


Tabela 2. Associação entre falta de envolvimento ativo do pai nos cuidados do filho e variáveis selecionadas. Porto Alegre, RS, 1998-2000.

\begin{tabular}{|c|c|c|c|c|c|c|}
\hline \multirow{3}{*}{ Variável } & \multicolumn{4}{|c|}{ Envolvimento do pai } & \multirow{3}{*}{$\mathrm{p}$} & \multirow{3}{*}{$\begin{array}{l}\text { Razão de prevalência } \\
\text { (IC 95\%)* }\end{array}$} \\
\hline & \multicolumn{2}{|c|}{ Inadequado $n=39$} & \multicolumn{2}{|c|}{ Ativo $\mathrm{n}=79$} & & \\
\hline & $\mathrm{n}$ & $\%$ & $\mathrm{n}$ & $\%$ & & \\
\hline Renda familiar <3 salários mínimos & 18 & 46,2 & 25 & 31,6 & 0,181 & $1,5(0,90 ; 2,48)$ \\
\hline Moradia precária** & 2 & 5,1 & 6 & 7,6 & 0.911 & $0,7(0,21 ; 2,54)$ \\
\hline Idade da mãe $<20$ anos & 10 & 25,6 & 12 & 15,2 & 0.263 & $1,5(0,87 ; 2,61)$ \\
\hline Idade do pai $<20$ anos & 4 & 10,3 & 4 & 5,1 & 0,505 & $1,6(0,75 ; 3,31)$ \\
\hline Cor da mãe não branca & 16 & 41,0 & 33 & 41,8 & 0,999 & $1,0(0,58 ; 1,65)$ \\
\hline Cor do pai não branca & 14 & 38,9 & 32 & 40,5 & 0,778 & $0,9(0,51 ; 1,50)$ \\
\hline Escolaridade da mãe $\leq 4$ anos & 13 & 33,3 & 12 & 15,2 & 0,042 & $1,9(1,13 ; 3,06)$ \\
\hline Escolaridade do pai $\leq 4$ anos & 14 & 35,9 & 11 & 13,9 & 0,012 & $2,1(1,29 ; 3,38)$ \\
\hline Mãe "do lar" & 36 & 92,3 & 60 & 75,9 & 0,058 & $2,8(0,93 ; 8,13)$ \\
\hline Pai sem emprego fixo & 11 & 28,2 & 15 & 19,0 & 0,368 & $1,4(0,81 ; 2,40)$ \\
\hline Criança do sexo feminino & 16 & 59,9 & 44 & 55,7 & 0,192 & $1,5(0,88 ; 2,52)$ \\
\hline Peso do criança ao nascer $<2.500 \mathrm{~g}$ & 5 & 12,8 & 5 & 6,3 & 0,401 & $1,6(0,80 ; 3,13)$ \\
\hline Criança não primogênita & 27 & 69,2 & 45 & 57,0 & 0,278 & $1,4(0,81 ; 2,54)$ \\
\hline Parto cesárea & 7 & 17,9 & 23 & 29,1 & 0,278 & $0,6(0,32 ; 1,30)$ \\
\hline Idade gestacional $<37$ semanas & 11 & 28,2 & 14 & 17,7 & 0,300 & $1,4(0,84 ; 2,48)$ \\
\hline Criança hospitalizada ao nascer & 4 & 10,3 & 13 & 16,5 & 0,483 & $0,7(0,27 ; 1,62)$ \\
\hline Pai não participou do pré-natal & 22 & 56,4 & 43 & 16,5 & 0,995 & $1,1(0,63 ; 1,80)$ \\
\hline Criança não recebia leite materno aos 4 meses & 11 & 28,2 & 27 & 34,2 & 0,657 & $0,8(0,46 ; 1,48)$ \\
\hline
\end{tabular}

* Teste qui-quadrado com correção de Yates

** Espaço pequeno, mal iluminado, sem forro ou assoalho, sem água encanada e/ou sanitário com descarga.

O envolvimento do pai nos cuidados com o lactente refere-se às tarefas que o pai desenvolve no dia-a-dia e que podem ser identificadas por qualquer pessoa. Contudo, a participação nas tarefas de cuidar do lactente não garante que isso seja feito com afeto e, portanto, tampouco, a qualidade do vínculo estabelecido.

Os resultados do presente estudo mostram que, nesta população urbana no Sul do Brasil, aos quatro meses de idade, $13 \%$ dos lactentes não tinham qualquer contato com o pai e $30 \%$ tinham pai que, apesar de coabitar, não se envolvia ativamente nos seus cuidados. Quando também foram incluídos na análise os pais que não coabitam com seus lactentes, a freqüência de não- envolvimento ativo com os filhos elevou-se a $48,3 \%$ da amostra.

Os resultados da análise de regressão de Poisson envolvendo todos os fatores de risco identificados na análise univariada como associados ao desfecho mostraram manutenção da associação de dois fatores de risco: a falta de envolvimento da mãe em trabalho externo ao lar e o casal apresentar problemas no seu relacionamento considerados de moderados a graves. Ainda que surpreenda a perda de significância de fatores como escolaridade, renda (que poderiam diminuir as condições do pai ser cuidador) e saúde mental (que pode prejudicar a disponibilidade emocional) ${ }^{19}$, os achados

Tabela 3. Associação entre níveis de saúde mental dos progenitores, dificuldades na relação conjugal e envolvimento ativo do pai nos cuidados com o filho. Porto Alegre, RS, 1998-2000.

\begin{tabular}{|c|c|c|c|c|c|c|}
\hline \multirow{3}{*}{ Variável } & \multicolumn{4}{|c|}{ Envolvimento do pai } & \multirow{3}{*}{$\mathrm{p}$} & \multirow{3}{*}{$\begin{array}{c}\text { Razão de } \\
\text { prevalência } \\
(\text { IC 95\%)* }\end{array}$} \\
\hline & \multicolumn{2}{|c|}{ Inadequado $\mathrm{n}=39$} & \multicolumn{2}{|c|}{ Ativo $\mathrm{n}=79$} & & \\
\hline & $\mathrm{n}$ & $\%$ & $\mathrm{n}$ & $\%$ & & \\
\hline \multicolumn{7}{|l|}{ Suspeita de transtorno mental** } \\
\hline Pai & 15 & 38,5 & 16 & 20,3 & 0,059 & $1,8(1,07 ; 2,90)$ \\
\hline Mãe & 20 & 51,3 & 24 & 30,4 & 0,027 & $1,8(1,07 ; 2,93)$ \\
\hline Dificuldades de moderadas a graves na relação conjugal*** & 17 & 43,6 & 11 & 13,9 & 0,000 & $2,5(1,55 ; 3,97)$ \\
\hline
\end{tabular}

\footnotetext{
* Teste qui-quadrado com correção de Yates

** Escala SRQ

*** Escala GARF
} 
Tabela 4. Análise de regressão de Poisson para avaliar a associação entre falta de envolvimento ativo do pai nos cuidados do filho e variáveis selecionadas. Porto Alegre, RS, 1998-2000.

\begin{tabular}{lccc}
\hline Variável & $\begin{array}{c}\text { Razão de Prevalência } \\
\text { Bruta (IC 95\%) }\end{array}$ & $\begin{array}{c}\text { Razão de Prevalência } \\
\text { Ajustada }(\text { IC 95\%) }\end{array}$ & $p$ \\
\hline Escolaridade do pai $\leq 4$ anos & $2,1(1,29 ; 3,38)$ & $1,1(0,93 ; 1,27)$ & 0,302 \\
Escolaridade da mãe $\leq 4$ anos & $1,9(1,13 ; 3,06)$ & $1,1(0,90 ; 1,23)$ & 0,515 \\
Mãe "do lar" & $2,8(0,93 ; 8,13)$ & $1,2(1,02 ; 1,39)$ & 0,028 \\
Renda familiar < 3 salários mínimos & $1,5(0,90 ; 2,48)$ & $1,0(0,84 ; 1,12)$ & 0,716 \\
Criança do sexo feminino & $1,0(0,40 ; 1,14)$ & $0,9(0,82 ; 1,04)$ & 0,218 \\
Pai com suspeita de transtorno mental* & $1,8(1,07 ; 2,90)$ & $1,1(0,95 ; 1,25)$ & 0,221 \\
Mãe com suspeita de transtorno mental* & $1,8(1,07 ; 2,93)$ & $1,0(0,90 ; 1,20)$ & 0,602 \\
Relação conjugal problemática** & $2,5(1,55 ; 3,97)$ & $1,2(1,00 ; 1,42)$ & 0,046 \\
\hline
\end{tabular}

* Escala SRQ

** Escala GARF

são coerentes. As expectativas sociais observadas na comunidade são caracterizadas por relações de gênero tradicionais, com divisão rígida de papéis entre os progenitores na qual à mulher são destinados os cuidados da casa e dos filhos enquanto o homem enfrenta o mundo externo, ocupando-se do sustento da família e dos valores e costumes da família., ${ }^{3,14}$

Há uma norma social na qual quando a mulher tem seu tempo dedicado a cuidar do filho, há menos necessidade e espaço para o envolvimento do pai. Por outro lado, como a cuidadora primária é a mulher, o fato de o homem estar em conflito com a mulher torna o acesso ao filho mais difícil para ele, pois a mãe solicitará ou facilitará o envolvimento entre pai e filho com menor freqüência. É possível inferir que se os casais com pouca participação do pai tivessem sido avaliados durante a gestação e no pós-parto, eles já apresentariam sinais de afastamento entre eles e estereotipia nos papéis que exercem na família. A delegação rígida de funções costuma ser produto de expectativas pessoais e familiares relacionadas com as regras sociais vigentes no grupo e/ou da distância emocional causada pelos conflitos crônicos do casal. A literatura apresenta dinâmica familiar semelhante em outras culturas. ${ }^{6,8,18}$

As mudanças que ocorrem na sociedade atual são decorrentes de modificação do perfil socioeconômico, expectativa média de vida, escolaridade, utilização de métodos contraceptivos, inserção da mulher no mercado de trabalho e ampliação dos direitos sociais que transformaram a estrutura e o funcionamento familiares. Essas modificações exigem uma ampliação do conhecimento dos profissionais sobre os fatores de risco e proteção nos novos arranjos familiares (famílias monoparentais e reconstituídas, pessoas que moram junto ou que moram em instituições) e as novas tendências nas famílias de haver maior envolvimento paterno desde o pré-natal, com participação ativa no parto e pós-parto. Assim, quando os homens participam ativamente da experiência do parto, passam a ter mais respeito e proximidade com suas mulheres. ${ }^{17}$
Em revisão de literatura brasileira entre 1990 e 1999, ${ }^{13}$ observou-se que o número de estudos sobre maternidade é aproximadamente três vezes maior do que sobre paternidade. Entretanto, estudos têm apontado que a presença atuante do homem na divisão de tarefas domésticas e no cuidado direto aos filhos favorece o aumento do bem-estar da mãe, expresso em relatos de experiências mais positivas sobre sua maternidade $\mathrm{e}^{6,22} \mathrm{e}$ também de evidências de melhor desenvolvimento da criança nas esferas cognitiva e social. , $^{1,71,21}$

Embora sem significância estatística, as variáveis baixa escolaridade e presença de transtorno mental paterno e materno mostraram razões de prevalência que devem ser levadas em consideração, pois são fatores usualmente considerados como associados com a qualidade da relação pais-filhos, denotando um prejuízo na relação pai-lactente. $^{19}$

Pela presente pesquisa pôde-se observar diretamente grupos familiares de todas as crianças nascidas num bairro em um determinado período e suas interações em seu meio ambiente. Além disso, utilizou o método de pontuação de instrumentos de pesquisa por consenso de dois observadores, minimizando, assim, possíveis vieses de aferição. Entretanto, é necessária cautela ao generalizar os achados do presente estudo, haja vista que foi realizado em uma única comunidade e em uma população não representativa de todos os estratos sociais.

Os achados do presente estudo indicam a necessidade de identificar precocemente transtornos da relação conjugal como forma de detectar a vulnerabilidade da relação pai-lactente. Os serviços de saúde devem ampliar o foco de observação e de cuidados para além da dupla mãe-lactente, promovendo condições para participação do pai nas consultas pré-natais e de puericultura e assim prevenir seu distanciamento do exercício do papel de pai, em consonância com as modificações no contexto familiar contemporâneo. 


\section{REFERÊNCIAS}

1. Aldous J, Mulligan GM. Father's child care and children's behavior problems. J Fam Issue. 2002;23(5):624-47. DOI: 10.1177/0192513X0202300 5003

2. Bowlby J. Apego. São Paulo: Martins Fontes; 1984.

3. Bustamante V. Participação paterna no cuidado da saúde de crianças pequenas: um estudo etnográfico com famílias de camadas populares. Cad Saude Publica. 2005;21(6):1865-74. DOI: 10.1590/S0102311X2005000600036

4. Falceto OG, Giugliani ER, Fernandes CL. Couples' relationships and breastfeeding: is there an association? J Hum Lact. 2004;20(1):46-55. DOI: $10.1177 / 0890334403261028$

5. Falceto OG, Giugliani ER, Fernandes CL. Influence of parental mental health on early termination of breastfeeding: a case-control study. J Am Board Fam Pract. 2004;17(3):173-83.

6. Feldman R. Parents' convergence on sharing and marital satisfaction, father involvement, and parentchild relationship at the transition to parenthood. Infant Mental Health J. 2000;21(3):176-91. DOI: 10.1002/1097-0355(200007)21:3<176::AIDIMHJ3>3.0.CO;2-4

7. Flouri E, Buchanan A. The role of father involvement in children's later mental health. J Adolesc. 2003;26(1):63-78. DOI: 10.1016/S01401971(02)00116-1

8. Gavin LE, Black MM, Minor S, Abel Y, Papas MA, Bentley ME. Young, disadvantaged fathers' involvement with their infants: an ecological perspective. J Adolesc Health. 2002;31(3):266-76. DOI: 10.1016/\$1054139X(02)00366-X

9. Glass J. Gender liberation, economic squeeze, or fear of strangers: why fathers provide infant care in dualearner families. J Marr Fam. 1998;60(4):821-34. DOI: $10.2307 / 353627$

10. Ip S, Chung M, Raman G, Chew P, Magula N, De Vine D. et al. Breastfeeding and maternal and infant health outcomes in developed countries. Evid Rep Technol Assess (Full Rep). 2007;(153):1-186.

11. Kaplan MD. The role of fathers. J Am Acad Child Adolesc Psych. 1996;35(6):699-700.

12. Keller WD, Hildebrandt KA, Richards ME. Effects of extended father-infant contact during the newborn period. Infant Behav Dev. 1985;8(3):337-50. DOI: 10.1016/0163-6383(85)90022-0
13. Levandowski DC, De Antoni C, Koller SH, Piccinini C. Paternidade na adolescência e os fatores de risco e de proteção para a violência na interação pai-criança. Interações. 2002;7(13):77-100.

14. Levandowski D, Piccinini CA. A interação pai-bebê entre adolescentes e adultos. Psicologia Reflexao e Critica. 2002;15(2):413-24. DOI: 10.1590/S010279722002000200018

15. Mari JJ, WilliamS P. A validity study of a psychiatric screening questionnaire (SRQ-20) in primary care in the city of São Paulo. Br J Psychiatry. 1986;148(1):23-6. DOI: 10.1192/bjp.148.1.23

16. Matthey S, Barnett B. Parent-infant classes in the early postpartum period: Need and participation by fathers and mothers. Infant Mental Health J. 1999;20(3):27890. DOI: 10.1002/(SICl)1097-0355(199923)20:3<278:: AID-IMHJ5>3.0.CO;2-I

17. Mazzieri SPM, Hoga LAK. Participação do pai no nascimento e parto: revisão da literatura. Rev Min Enf. 2006;10(2):166-70.

18. McHale JP, Kazali C, Rotman T, Talbot J, Carleton M, Lieberson R. The transition to coparenthood: parents' prebirth expectations and early coparental adjustment at 3 months postpartum. Dev Psychopathol. 2004;16(3):711-33. DOI: $10.1017 /$ S0954579404004742

19. Paulson JF, Dauber S, Leiferman JA. Individual and combined effects of postpartum depression in mothers and fathers on parenting behavior. Pediatrics. 2006;118(2):659-68. DOI: 10.1542/peds.2005-2948

20. Pedersen FA, Suwalsky JT, Cain RL, Zaslow MJ, Rabinovich BA. Paternal care of infants during maternal separations: associations with father-infant interaction at one year. Psychiatry. 1987;50(3):193205.

21. Ramos S de P. What can we learn from psychoanalysis and prospective studies about chemically dependent patients? Int J Psychoanal. 2004;85(Pt 2):467-88. DOI: 10.1516/D7UK-ULMK-AT01-MYCQ

22. Rogers SJ, White LK. Satisfaction with parenting: the role of marital happiness, family structure, and parents' gender. J Marri Fam. 1998;60(2):293-308. DOI: $10.2307 / 353849$

23. Seward RR, Yeatts DE, Zottarelli LK. Parental leave and father involvement in child care: Sweden and the United States. J Comp Fam Stud. 2002;33(3):387-99 